\title{
Nonstochastic Behavior of Atomic Surface Diffusion on Cu(111) down to Low Temperatures
}

\author{
J. Ferrón \\ Grupo de Física INTEC-FIQ, CONICET, Universidad Nacional del Litoral, 3000-Santa Fe, Argentina \\ L. Gómez \\ Facultad de Ciencias Exactas, Ingeniería y Agrimensura, Instituto de Física Rosario, 2000-Rosario, Argentina \\ J. J. de Miguel and R. Miranda \\ Departamento de Física de la Materia Condensada and Instituto de Ciencia de Materiales "Nicolás Cabrera"; \\ Universidad Autónoma de Madrid, Cantoblanco, 28049-Madrid, Spain \\ (Received 22 March 2004; published 15 October 2004)
}

\begin{abstract}
Atomic diffusion is usually understood as a succession of random, independent displacements of an adatom over the surface's potential energy landscape. Nevertheless, an analysis of molecular dynamics simulations of self-diffusion on $\mathrm{Cu}(111)$ demonstrates the existence of different types of correlations in the atomic jumps at all temperatures. Thus, the atomic displacements cannot be correctly described in terms of a random walk model. This fact has a profound impact on the determination and interpretation of diffusion coefficients and activation barriers.
\end{abstract}

DOI: 10.1103/PhysRevLett.93.166107

PACS numbers: 68.35.Fx, 68.47.De, 71.15.Pd, 82.20.Db

Surface diffusion is a most influential process at the atomic scale, lying at the core of many relevant fields. In catalysis and surface chemistry the ability of the reactants to come together and/or reach the active surface sites is the first factor controlling the reaction rates [1]. Likewise, in epitaxial growth the adatom mobility decisively influences the nucleation probability and the average distance and size of the objects (islands) formed [2]; kinetic limitations result in the accumulation of roughness as growth proceeds [3].

Despite the many efforts devoted to studying this phenomenon, our current understanding is far from being complete [4]. Continuous advancements in both theory and experiments are unveiling a rich phenomenology unsuspected till now; even for the simplest self-diffusion case, new basic mechanisms are still being discovered. First it was site exchange, predicted theoretically [5] and then detected in field ion microscopy experiments [6]. Later, evidence was found on the existence of "long jumps," i.e., atomic displacements spanning several lattice constants [7,8], lifting the restriction to nearestneighbor hops. The last finding so far is subsurface diffusion, in which adatoms move below a loosely bound overlayer deposited on a given surface $[9,10]$.

Diffusion is usually described in terms of atomic hops from one adsorption site to a nearby one. The rate of successful jumps between two equilibrium positions separated by an energy barrier $E_{m}$ is given by

$$
\gamma=\gamma_{0} \exp \left(-E_{m} / k_{B} T\right) \text {. }
$$

Assuming that the diffusing adatom performs a random walk, and applying transition state theory (TST), the hopping rate can be related to the diffusion coefficient $[11,12]$

$$
D_{\gamma}=D_{0}(T) \exp \left(-E_{m} / k_{B} T\right)=\gamma l^{2} / 4
$$

$l$ being the hop length [1.47 $\AA$ for $\mathrm{Cu}(111)]$. $D_{0}$ is slightly temperature dependent, but it can be safely taken as a constant [12,13]. Alternatively, the diffusion coefficient $D_{\sigma}$ can be obtained from the adatom displacements through the Einstein relation in two dimensions

$$
\sigma^{2}(t)=\frac{1}{N}\left[\sum_{i=1}^{N}\left(x_{i}(t)-x_{0}\right)^{2}+\left(y_{i}(t)-y_{0}\right)^{2}\right]=4 D_{\sigma} t,
$$

where $x_{i}(t), y_{i}(t)$ are the surface coordinates at time $t$ for $N$ different initial conditions. Provided that all the relevant processes are taken into account, and that the assumptions of TST are valid for all of them, these two formulations should be equivalent and $D_{\gamma}=D_{\sigma}$.

These conditions are not always fulfilled. When the thermal energy is higher than the diffusion barrier $\left(k_{B} T \geq E_{m}\right)$ the adatoms move rather freely over the surface and the definition of a hopping frequency loses sense. It is generally accepted that at temperatures of the order of $E_{m} / 2 k_{B}$ most of the assumptions of TST fail, diffusion cannot be represented by a random walk anymore and long, correlated jumps become more and more important [14]. Hence at room temperature (RT, $300 \mathrm{~K}$ ), an $E_{m}$ not smaller than $50 \mathrm{meV}$ is required to satisfy the above condition. For compact metallic faces such as $\mathrm{Cu}(111)$ the activation energy for monomer diffusion can be clearly smaller $[15,16]$; therefore TST would not apply and correlated displacements are to be expected.

In this work, we demonstrate the existence of other correlated atomic movements even at low temperature, when single hops predominate, thus questioning many 
assumptions accepted so far; we also explore the relationship between the activation barriers determined from the simulations and those calculated statically. We draw our conclusions from analyzing the outcome of molecular dynamics (MD) simulations for self-diffusion of $\mathrm{Cu}$ on $\mathrm{Cu}(111)$ in which we follow the displacement of an adatom over a fully relaxed surface at different temperatures. Our MD code [17] uses interatomic potentials based on the embedded atom model (EAM) [18]. The sample was a slab of 14 layers with 270 atoms in each, and vacuum on both sides; periodic boundary conditions were used in all directions. The three bottom layers were frozen to simulate the bulk. The evolution of a single adatom at the upper surface was followed for different temperatures. In order to obtain reliable statistics, the simulation was extended for up to $10 \mathrm{~ns}$ for the lower temperatures; similar numbers of jumps (with shorter simulation times) were accumulated at high temperatures. For the analysis of the atomic trajectories, we split the surface into fcc and hcp cells, and detect the jumps automatically by the passage of the diffusing atom from one region to another, as illustrated by the inset in Fig. 1; the time elapsed between two consecutive jumps is the atom's residence time at that position.

In the MD simulation the mean square displacement $\sigma^{2}(t)$ of the diffusing particle can be obtained independently of $\gamma$. The latter is calculated as the number of successful jumps divided by the total elapsed time. In Fig. 1 we depict in the usual form of an Arrhenius plot the values of the diffusion coefficient $D_{\sigma}$ obtained from the mean square displacement, and that found from the analysis of the hopping frequencies $\left(D_{\gamma}\right)$. From a fit to

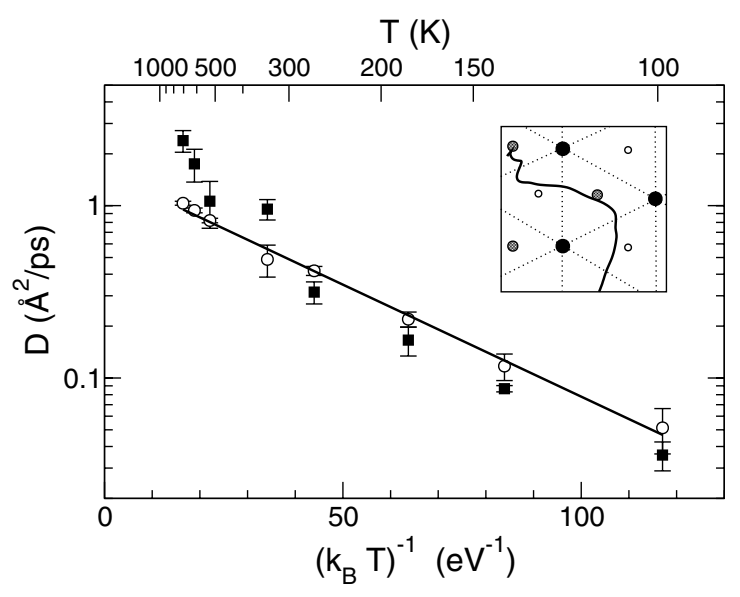

FIG. 1. Diffusion coefficient for $\mathrm{Cu}$ atoms on $\mathrm{Cu}(111)$, calculated either by measuring the atomic displacement as a function of time $\left(D_{\sigma}\right.$, solid squares) or from the hopping frequency ( $D_{\gamma}$, open circles). The solid line is an Arrhenius fit to $D_{\gamma}$, with $D_{0}=1.56 \times 10^{-4} \mathrm{~cm}^{2} \mathrm{~s}^{-1}$ and $E_{m}=30 \pm 1 \mathrm{meV}$. The inset illustrates the definition of the surface cells with a typical ballistic trajectory and the determination of residence times: the solid circles are surface atoms, while the smaller gray and open dots mark the hcp and fcc sites, respectively. the latter, which displays a more Arrhenius-like behavior, an activation energy of $30 \pm 1 \mathrm{meV}$ is found (solid line in Fig. 1); fits to $D_{\sigma}$ yield values similar to this one but only in the low temperature range. Above $\sim 300 \mathrm{~K}$, which corresponds roughly to the $E_{m} / 2 k_{B}$ condition enunciated above, $D_{\sigma}$ clearly deviates from the Arrhenius law toward higher values. A similar phenomenon was already observed in simulations reported by Kallinteris et al. [19], who ascribed this behavior to the onset of a new activated mechanism, namely, the diffusion along the [110] direction by means of double jumps. Our simulations reveal that the increasing slope of $D_{\sigma}$ is due to the predominance of the long trajects, which cause net atomic displacements much larger than what could be expected from a random walk.

Figure 2 shows some representative atomic trajectories at different temperatures. In general they consist of displacements, which frequently extend up to several lattice sites, separated by periods of vibration within a single surface cell; the relative abundance of each type of event depends on the temperature. Similar trajectories have been reported previously, resulting from simulations with other sets of interatomic potentials [20] and also in experiments [21]. Evidently, adatom diffusion at high temperature can hardly be considered a random phenomenon; any thermally activated process, characterized by an attempt frequency (preexponential factor) and an energy barrier, should have the same occurrence probability for all equivalent paths. Quite on the contrary, clearly deterministic trajectories can be observed in Fig. 2(c); the path followed by the $\mathrm{Cu}$ adatom is reminiscent of surface channeling, where an energetic diffusing particle is steered along its trajectory by the potential accounting for its interactions with the surrounding atoms. The enhanced diffusivity at high temperature, above the expected Arrhenius behavior, is thus caused by these correlated movements. On the other hand, at low temperatures random processes based on single jumps separated by long stays at a given adsorption well seem to be dominant and no preferred trajectories are evident.

A detailed study of the atomic trajectories demonstrates that this is not the case. Figure 3 shows histograms depicting the statistical distribution of residence times for different temperatures. The appearance of large peaks at very short times is evidence for the lack of randomness in

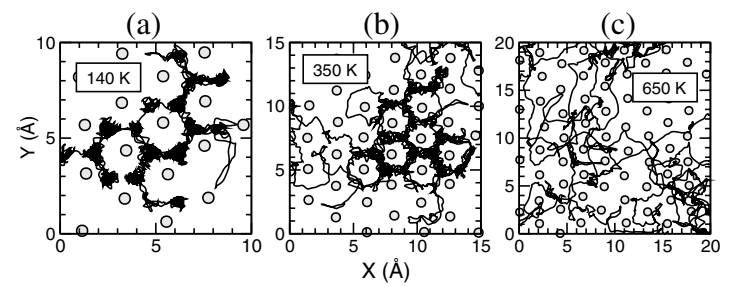

FIG. 2. Some typical examples of atomic trajectories of $\mathrm{Cu}$ adatoms self-diffusing on $\mathrm{Cu}(111)$ at diverse temperatures. Notice the different sizes of the regions exposed. 


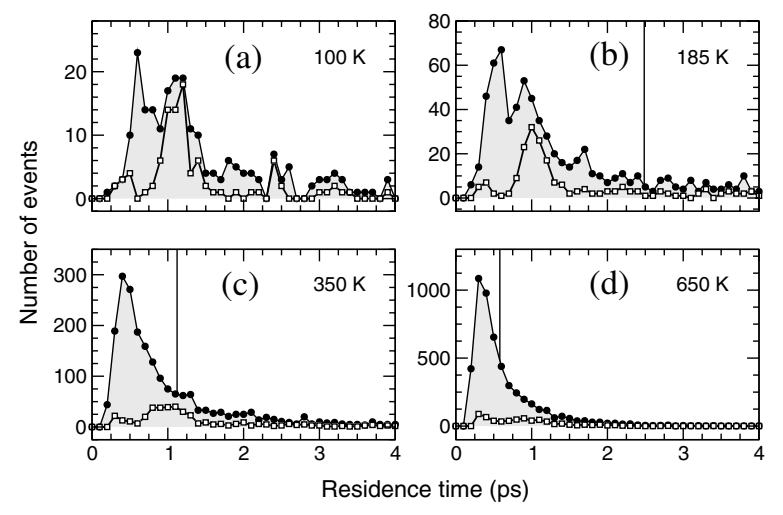

FIG. 3. Statistical distribution of residence times of the diffusing $\mathrm{Cu}$ monomers as a function of substrate temperature. Solid circles: all kinds of jumps; open squares: recrossings. The vertical lines mark the average residence time at each particular temperature; at $100 \mathrm{~K}$ the corresponding value of $10.6 \mathrm{ps}$ falls outside the graph range.

the diffusion process. The average residence time for each substrate temperature is marked by the vertical line in those graphs. The shift to lower times (i.e., higher jump frequencies) is clearly due to the disappearance of several types of processes, most significantly the recrossings, with their longer associated times, and not to a larger jump probability, as would be for a thermally activated process.

The stochastic nature of diffusion can be analyzed in full detail by sorting out the different kinds of hops. In Fig. 3, the solid circles take into account all the jumps detected in our simulations. A striking feature is the appearance of a double peak at short residence times for the lower temperatures. The first one corresponds to what we call ballistic jumps: rapid crossings making up the long displacements in which the adatom traverses several surface cells in a single impulse. This is obviously the shortest residence time observed and is related to the average velocity of the diffusing adatoms. We shall call this elementary time interval $\tau_{0}$; its magnitude decreases with increasing substrate temperature, from $0.6 \mathrm{ps}$ at $100 \mathrm{~K}$ down to $0.38 \mathrm{ps}$ at $650 \mathrm{~K}$, reflecting the higher kinetic energy of the adatoms.

The curves marked with open squares in Fig. 3 depict the times associated with recrossing events [22], that is, two consecutive jumps that bring the adatom back to its former position. At $100 \mathrm{~K}$ this kind of processes completely accounts for the second peak in the general distribution. Significantly, this peak is centered at a time close to $2 \tau_{0}$. Below $185 \mathrm{~K}$ yet another clear peak can be seen at about $3 \tau_{0}$. As expected, this triple transition time appears after two frustrated jump attempts. Quite surprisingly, almost none of these hops is a recrossing, as demonstrated by the statistics in Figs. 3(a) and 3(b); $\frac{1}{3}$ of them would be expected if the process were truly random. We shall call these kinds of jumps "double bounces" for brevity.
Our statistical analysis of diffusion jumps is presented in Fig. 4. First, it is remarkable the large number of ballistic jumps observed even at low temperature; needless to say, they absolutely dominate above $300 \mathrm{~K}$. At low temperature the recrossings are more frequent than expected ( $\frac{1}{3}$ of the total) for a random process. This is probably due to the geometric arrangement of atoms in the (111) face: The adatom jumping through the saddle point is directed in a collision trajectory toward the third atom in the threefold cell. If the energy is not enough to set the channeling effect in, the atom is backscattered preferentially in a recrossing trajectory. Our results show that these processes occur before the adatom becomes thermalized again. Neither of them can be considered stochastic; rather, this behavior hints toward some participation of the substrate atoms. As for the double bounces, their topological analysis is also shown in Fig. 4(b): despite the rise at about $200 \mathrm{~K}$, the percentage of them that are recrossings never reaches $\frac{1}{3}$, implying that the choice of directions is also nonrandom. We thus conclude that at least three oscillations within an adsorption well, or a residence time $\geq 4 \tau_{0}$, are required for thermalization.

In summary, we find evidence in our simulations showing that most of the atomic jumps in surface diffusion are biased, i.e., the process is not stochastic: the correlated jumps exceed $95 \%$ of the total above $500 \mathrm{~K}$ and even at $100 \mathrm{~K}$ amount to more than $50 \%$. The kind of correlations, however, is not the same. The natural question is how this can influence the determination of the activation

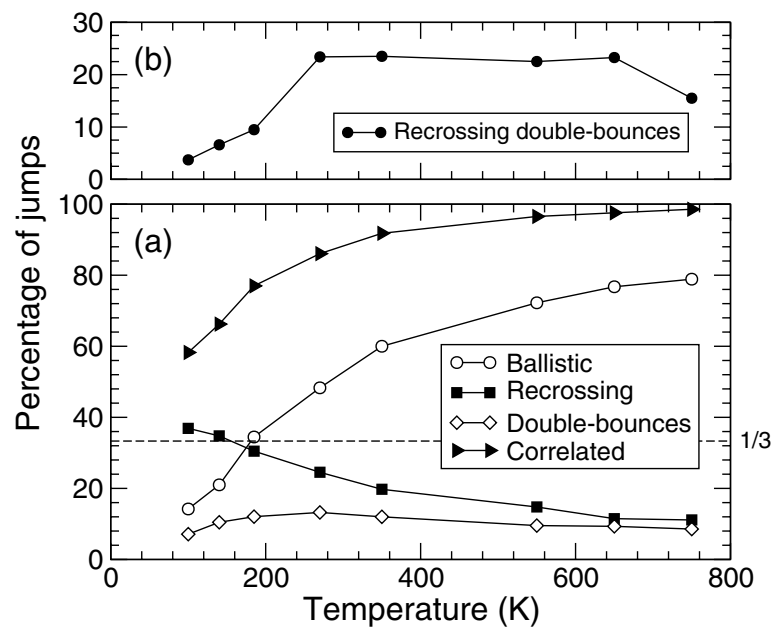

FIG. 4. Statistical analysis of correlations in atomic jumps. (a) Percentage with respect to the total of: ballistic or long jumps (open circles), recrossings (squares) and "double bounces" (diamonds). The dotted line marks the $1 / 3$ expected probability of recrossings for random hops. (b) Percentage of "double bounces" (jumps after a residence time $3 \tau_{0}$ ) that return to the previous adsorption site. The total fraction of correlated jumps, after our analysis, is given by the solid triangles in (a); even at $100 \mathrm{~K}$ this figure exceeds $50 \%$, reaching nearly $100 \%$ at the higher temperatures. 


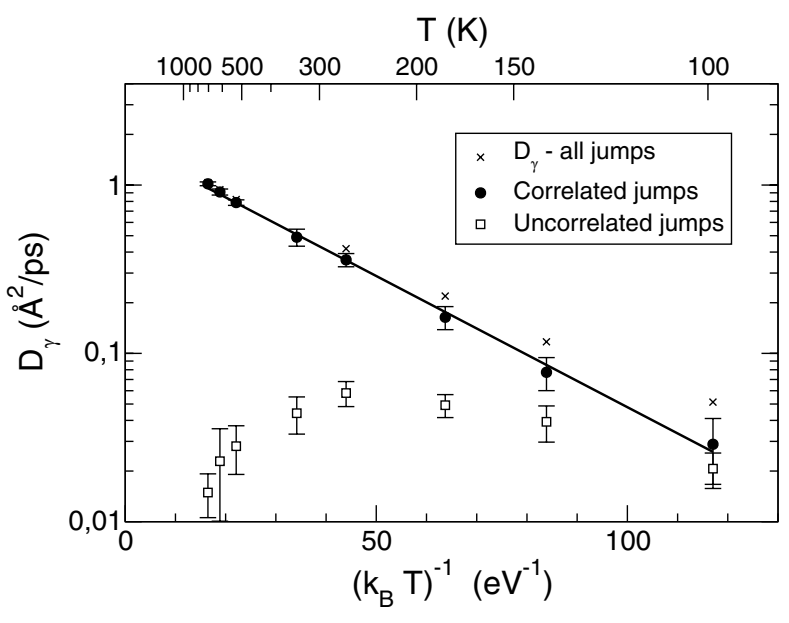

FIG. 5. Contributions to the diffusion coefficient $D_{\gamma}$ obtained taking separately the correlated jumps (solid circles) and the uncorrelated ones (open squares). The solid line is an Arrhenius fit to the former, yielding $D_{0}=1.73 \times$ $10^{-4} \mathrm{~cm}^{2} \mathrm{~s}^{-1}$ and $E_{m}=36 \pm 2 \mathrm{meV}$. The crosses are the uncorrected $D \gamma$ values from Fig. 1, shown for comparison.

energies for surface diffusion. Although there is some dispersion in the literature $[15,16]$ there seems to be some recent coincidence on fixing the diffusion energy for $\mathrm{Cu}$ monomers on $\mathrm{Cu}(111)$ between 40 [12,19,23,24] and $50 \mathrm{meV}$ [25]. Our own calculations using static relaxation with the same set of potentials as in the MD simulations yield $40 \pm 6 \mathrm{meV}$. Nevertheless, the fit to $D_{\gamma}$ in Fig. 1 yields only $30 \mathrm{meV}$. In order to get some insight into this discrepancy, we have plotted separately in Fig. 5 the contributions to the diffusion coefficient $D_{\gamma}$ from correlated (solid circles) and uncorrelated jumps (open squares). The correlated processes are much more abundant, and for this reason their statistics is better. At high temperature the number of uncorrelated events is very low, and therefore the difference with the values of $D_{\gamma}$ obtained using all jumps (crosses, same data of Fig. 1) is negligible. On the other hand, most of the uncorrelated jumps occur at low temperature, and for this reason the contribution of the correlated ones at low temperature is smaller. This results in a higher slope in the Arrhenius fit (solid line in Fig. 5) of $36 \pm 2 \mathrm{meV}$, in good agreement with the theoretical barriers. Since the calculations of static barriers involve relaxations of all neighboring atoms, it is tempting to link the origin of the adatom's correlated displacements to its interactions with them. Obviously, the key processes here are the rates of energy transfer between the substrate and the adatom, and the achievement of thermal equilibrium by the latter. Further work is in progress to study these subjects in detail. As for the experimental measurements of activation energies, they are usually based on atomic displacements rather than on hopping frequencies: if the experiments are done at or above $300 \mathrm{~K}$, one can expect to be in the range where ballistic jumps dominate, overestimating the dif- fusion coefficient and yielding higher slopes (i.e., activation energies) as demonstrated by the data in Fig. 1. All these subtleties should be kept in mind, as they could explain many of the discrepancies frequently found in the literature [26], particularly when comparing theoretical and experimental values of diffusion coefficients and migration energies.

This work has been financed by the CICyT through Project No. BFM2001-0174 and by CONICET through PIP 2553/99. J. F. thanks Fundación Antorchas for financial support.

[1] V. P. Zhdanov and B. Kasemo, Surf. Sci. Rep. 20, 113 (1994).

[2] See, for instance, Surface Diffusion, Atomistic and Collective Processes, edited by M.C. Tringides, NATO Advanced Study Institutes, Ser. B, Vol. 360 (Plenum, New York, 1997).

[3] Z. Zhang, J. Detch, and H. Metiu, Phys. Rev. B 48, 4972 (1993).

[4] J.V. Barth, Surf. Sci. Rep. 40, 75 (2000).

[5] G. DeLorenzi and G. Jacucci, Surf. Sci. 164, 526 (1985).

[6] G. L. Kellogg and P. J. Feibelman, Phys. Rev. Lett. 64, 3143 (1990); C. Chen and T. T. Tsong, Phys. Rev. Lett. 64, 3147 (1990).

[7] S. C. Wang, J. D. Wrigley, and G. Ehrlich, J. Chem. Phys. 91, 5087 (1989).

[8] G. Antczak and G. Ehrlich, Phys. Rev. Lett. 92, 166105 (2004).

[9] J. Camarero et al., Phys. Rev. Lett. 81, 850 (1998).

[10] J. Neugebauer et al., Phys. Rev. Lett. 90, 056101 (2003).

[11] S. Glasstone, K. J. Laidler, and H. Eyring, The Theory of Rate Processes (McGraw-Hill, New York, 1941).

[12] U. Kürpick, Phys. Rev. B 64, 075418 (2001).

[13] U. Kürpick, A. Kara, and T. S. Rahman, Phys. Rev. Lett. 78, 1086 (1997).

[14] G. DeLorenzi, G. Jacucci, and V. Pontikis, Surf. Sci. 116, 391 (1982)

[15] P. Stoltze, J. Phys. Condens. Matter 6, 9495 (1994).

[16] M. Karimi, T. Tomkowski, G. Vidali, and O. Biham, Phys. Rev. B 52, 5364 (1995).

[17] DYNAMO code released by M. S. Daw and M. I. Baskes.

[18] S. M. Foiles, M. I. Baskes, and M. S. Daw, Phys. Rev. B 33, 7983 (1986).

[19] G. G. Kallinteris, G. A. Evangelakis, and N. I. Papanicolaou, Surf. Sci. 369, 185 (1996).

[20] F. Montalenti and R. Ferrando, Phys. Rev. B 58, 3617 (1998).

[21] D. C. Senft and G. Ehrlich, Phys. Rev. Lett. 74, 294 (1995).

[22] D. E. Sanders and A. E. DePristo, Surf. Sci. Lett. 264, L169 (1992)

[23] J. Repp et al., Phys. Rev. Lett. 85, 2981 (2001).

[24] N. Knorr et al., Phys. Rev. B 65, 115420 (2002).

[25] A. Bogicevic et al., Phys. Rev. Lett. 85, 1910 (2000).

[26] Z. Zhang, K. Haug, and H. Metiu, J. Chem. Phys. 93, 3614 (1990). 